\title{
An Alternative Origin for Hypervelocity Stars
}

\author{
Mario G. Abadi \\ Instituto de Astronomía Teórica y Experimental (IATE) \\ Observatorio Astronómico de Córdoba and CONICET \\ Laprida 854 X5000BGR Córdoba, Argentina \\ mario@oac.uncor.edu \\ Julio F. Navarro \\ Department of Physics and Astronomy, University of Victoria \\ 3800 Finnerty Road, Victoria, BC V8P 5C2, Canada \\ jfn@uvic.ca \\ and \\ Matthias Steinmetz \\ Astrophysikalisches Institut Potsdam \\ An der Sternwarte 16, 14482 Potsdam, Germany \\ msteinmetz@aip.de
}

\begin{abstract}
Halo stars with unusually high radial velocity ("hypervelocity" stars, or HVS) are thought to be stars unbound to the Milky Way that originate from the gravitational interaction of stellar systems with the supermassive black hole at the Galactic center. We examine the latest HVS compilation and find peculiarities that are unexpected in this black hole-ejection scenario. For example, a large fraction of HVS cluster around the constellation of Leo and share a common travel time of $\sim 100-200$ Myr. Furthermore, their velocities are not really extreme if, as suggested by recent galaxy formation models, the Milky Way is embedded within a $2.5 \times 10^{12} h^{-1} M_{\odot}$ dark halo with virial velocity of $\sim 220 \mathrm{~km} / \mathrm{s}$. In this case, the escape velocity at $\sim 50 \mathrm{kpc}$ would be $\sim 600 \mathrm{~km} / \mathrm{s}$ and very few HVS would be truly unbound. We use numerical simulations to show that disrupting dwarf galaxies may contribute halo stars with velocities up to and sometimes exceeding the nominal escape speed of the system. These stars are arranged in a thinly-collimated outgoing "tidal tail" stripped from the dwarf during its latest pericentric passage. We speculate that some HVS may therefore be tidal debris from a dwarf recently disrupted near the center of the Galaxy. In this interpretation, the angular clustering of HVS results because from our perspective the tail is seen nearly "end on", whereas the common travel time simply reflects the fact that these stars were stripped simultaneously from the dwarf during a single pericentric passage. This proposal is eminently falsifiable, since it makes a number of predictions that are distinct from the black-hole ejection mechanism and that should be testable with improved HVS datasets.
\end{abstract}

Subject headings: Galaxy: disk - Galaxy: formation - Galaxy: kinematics and dynamics - Galaxy: structure 


\section{Introduction}

The existence of ultra high-speed stars in the halo of the Milky Way was recognized by Hills (1998) as an inevitable consequence of the presence of a supermassive black hole $(\mathrm{SMBH})$ in a region of high stellar density such as the Galactic center. Hills estimated that the disruption of tightly bound binaries in the vicinity of the black hole may propel stars to speeds exceeding 1000 $\mathrm{km} / \mathrm{s}$, well beyond the escape velocity from the Milky Way. The discovery of a population of "hypervelocity" stars (HVS, for short, Brown et al. 2005) in radial velocity surveys of faint B-type stars in the Galactic halo (Brown et al. 2006, 2007a,b) gave strong support to Hills' proposal and led to substantial theoretical interest in the topic. The latest compilation by Brown, Geller \& Kenyon (2008) lists 16 HVS, all receding at speeds exceeding $\sim 300 \mathrm{~km} / \mathrm{s}$ and reaching up to $\sim 720$ $\mathrm{km} / \mathrm{s}$ in the Galactic rest frame.

HVS are widely thought to owe their extreme velocities to Hills' mechanism, since many of the stars appear to be short-lived, massive main sequence stars such as those often found near the Galactic center. Other mechanisms also capable of accelerating stars to high speeds, such as binary disruption in stellar clusters, have usually been disfavored on the grounds that the maximum ejection velocity is unlikely to exceed the escape velocity at the surface of a star, and thus would only be able to accelerate stars up to no more than a few hundred $\mathrm{km} / \mathrm{s}$. The mediation of the much deeper potential well of a supermassive black hole thus appears needed to explain the extreme velocities observed for the HVS population.

Although some HVS have very likely been ejected by the Galactic SMBH, there are a number of issues that remain unresolved in this scenario. For example, the velocity distribution of HVS and, in particular, the lack of very high-velocity stars (i.e., with speeds exceeding $1000 \mathrm{~km} / \mathrm{s}$ ), is not easily understood (see, e.g., Sesana, Haardt \& Madau 2007). Nor is the existence of at least one HVS moving at a speed greater than $600 \mathrm{~km} / \mathrm{s}$ in the Galactic rest frame (HD 271791; Heber et al 2008) whose proper motion apparently rules out a Galactic center origin.

Although small number statistics may explain away these concerns, we examine in this Letter further peculiarities in the spatial distribution and kinematics of HVS and argue that these suggest, at least for some HVS, an origin different from the SMBH-ejection mechanism. We begin by examining the distribution of travel times and the angular clustering of HVS compiled from the literature, and use numerical simulations to explore an alternative mechanism able to populate the Galactic halo with high-speed stars. We end by considering several observational tests that may, in the future, be used to discriminate between these competing scenarios.

\section{Analysis}

\subsection{Observational data}

We use primarily data from the Brown et al (2006, 2007a,b, hereafter BGKK, for short) MMT and Whipple surveys of B-type halo stars selected from the Sloan Digital Sky Survey. Our analysis uses Galactic rest-frame radial velocities, positions in the sky, and distances, as given by the authors. We note that distance estimates are only listed for stars with velocities exceeding $\sim 280 \mathrm{~km} / \mathrm{s}$. These estimates typically assume that the stars are in the main sequence, although it is difficult to rule out that some (or many) are evolved stars in the blue horizontal branch (BHB). This is one of the main systematic uncertainties in our analysis, but it is a shortcoming shared with all other attempts to study the kinematics of HVS stars with current datasets.

\subsection{Travel times}

The left panel in Figure 1 shows the Galactic rest frame radial velocity distribution of all stars in the BGKK survey, whereas the right panel shows the radial velocity of all stars with published distance estimates. These are all high-speed stars $\left(V_{r} \gtrsim 280 \mathrm{~km} / \mathrm{s}\right)$ out in the tail of the radial velocity distribution (filled symbols). Open symbols correspond to the 10 new stars recently reported by Brown, Geller \& Kenyon (2008, hereafter BGK08) and are added here only for completeness, since data for the underlying survey from which these stars have been identified are not yet publicly available. ("Dotted" symbols identify stars in the circle shown in Fig. 2 as discussed below.) For illustration, we have added in Figure 1 


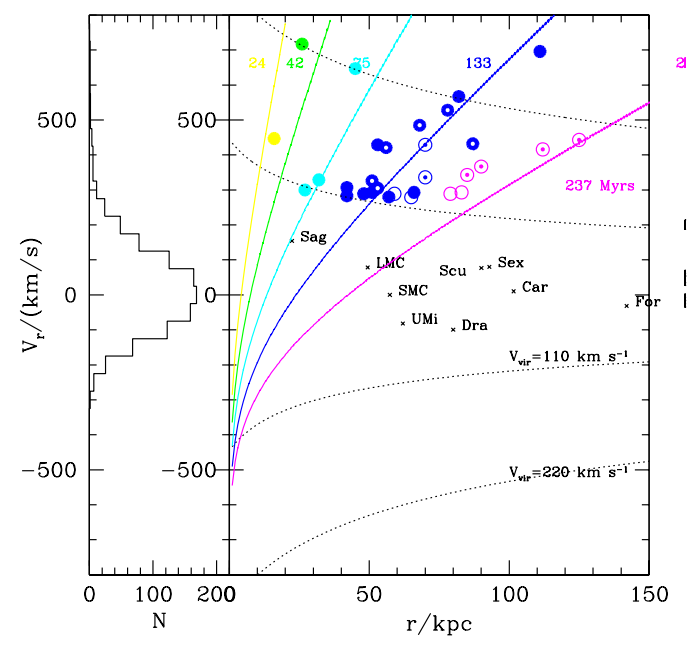

Fig. 1.- Galactocentric rest frame radial velocity versus distance for all high-velocity stars with estimated distances compiled from the BGKK (filled symbols) and BGK08 (open symbols) references. Symbols with central dots correspond to stars that fall in the Leo I-centered circle shown in Fig. 2 Solid lines show travel times from the Galactic center in Myrs, as indicated by labels, computed using the Galactic potential of Bromley et al. (2006). Dotted curves show the escape velocity of an NFW (Navarro, Frenk \& White 1996, 1997) halo with virial velocity $V_{200}=220 \mathrm{~km} / \mathrm{s}$ (the circular velocity at the solar circle) and $110 \mathrm{~km} / \mathrm{s}$ (half of that value), for reference. Note that for $V_{200}=220$ $\mathrm{km} / \mathrm{s}$ only one HVS is clearly unbound to the Galaxy. The left panel shows the radial velocity histogram for all stars in the BGKK dataset. Some Milky Way satellites are also included for comparison.

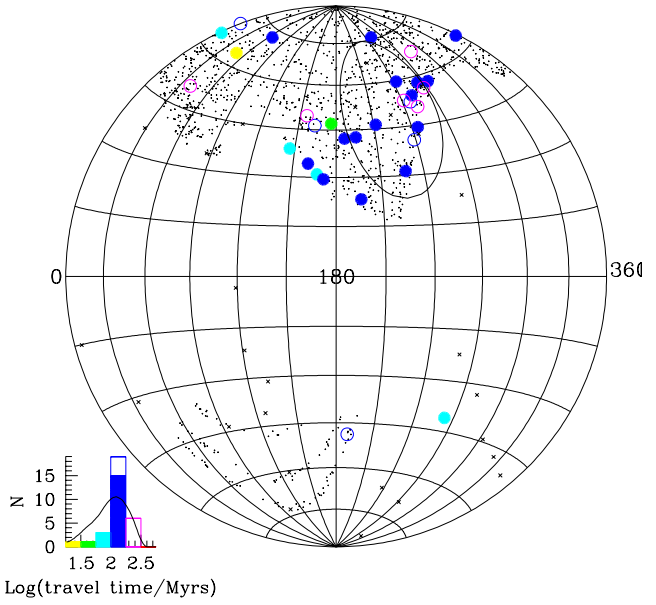

Fig. 2.- Aitoff projection in Galactic coordinates for all stars in the BGKK survey. Stars highlighted in color are high-speed $\left(V_{r} \gtrsim 300 \mathrm{~km} / \mathrm{s}\right)$ stars with estimated distances (filled circles), and include a few new stars reported by BGK08 (open symbols). The color encodes the travel time of each star, as shown by the histogram in the bottom left inset. The filled histogram corresponds to BGKK stars, the open histogram adds the new stars in the BGK08 sample. The solid curve shows the travel-time distribution predicted by a model where stars are continuously ejected by the SMBH at the Galactic center (Bromley et al. 2006), after taking into account the finite lifetime of the stars and the effective survey volume. The black circle in the aitoff panel delineates a $26^{\circ}$-diameter region in the constellation of Leo. More than half of all HVS with $V_{r}>350 \mathrm{~km} / \mathrm{s}$ are contained within that region. This represents a significant overdensity of HVS in the direction of Leo, since the same region contains only about $\sim 20 \%$ of all stars in the survey. A few other Milky Way satellites are shown for reference. 
the position in the $r-V_{r}$ plane of several Milky Way satellite companions, as labeled.

For each star with published distance estimate and radial velocity we compute a travel time from the Galactic center, assuming purely radial orbits in the simple Galactic potential model of Bromley et al (2006). This is given by a spherically symmetric density profile, $\rho(r)=\rho_{0} /\left(1+\left(r / r_{c}\right)^{2}\right)$, where $\rho_{0}=1.396 \times 10^{4} M_{\odot} \mathrm{pc}^{-3}$ and $r_{c}=8 \mathrm{pc}$ (Kenyon et al 2008). The thick solid curves in Fig. 1 represent the loci of stars with constant travel time (labeled in Myr), whereas the dotted lines show the escape velocity from the Milky Way, assuming it is embedded within a cold dark matter halo with virial velocity $11 V_{200}=220 \mathrm{~km} / \mathrm{s}$ and $110 \mathrm{~km} / \mathrm{s}$, respectively. The HVS data in Figure 1is colored according to travel time, binned in equally spaced logarithmic intervals (see also the histogram in the inset of Fig. (2).

Figure 11 illustrates a few interesting points. One is that very few stars are actually unbound if the virial velocity of the Milky Way is of the order of $220 \mathrm{~km} / \mathrm{s}$, the circular speed at the solar circle. This is actually what is required by recent models of galaxy formation in order to match simultaneously the zero-point of the Tully-Fisher relation and the normalization of the galaxy luminosity function (see, e.g., Croton et al 2006 for a full discussion). In other words, for $V_{200}=220$ $\mathrm{km} / \mathrm{s}$, the HVS speeds are unusually high, but not necessarily extreme, and only one HVS would be clearly unbound. This is important, since it suggests that more prosaic dynamical effects that do not rely on SMBH ejection may be responsible for the unusual speeds of the HVS population.

The second point to note is that the distribution of HVS travel times is not uniform. This is illustrated in the histogram shown in the inset of Fig. 2, 19 of the 30 HVS in the northern hemisphere seem to share a common travel time (see bin centered at $\sim 133 \mathrm{~km} / \mathrm{s}$ ). The peak in the histogram is higher than expected from a model

\footnotetext{
${ }^{1}$ We assume a Navarro, Frenk \& White $(1996,1997)$ mass profile for the halo (with concentration $c=15$ ) and define the virial velocity as the circular velocity within a radius, $r_{200}$, enclosing a mean overdensity of 200 times the critical value for closure. The virial velocity defines implicitly the halo mass: for $V_{200}=220 \mathrm{~km} / \mathrm{s}$, the mass within $r_{200}=$ $220 \mathrm{kpc} / \mathrm{h}$, is $M_{200}=2.5 \times 10^{12} M_{\odot} / \mathrm{h}$. We assume $\mathrm{h}=0.73$ throughout the paper.
}

where ejections occur uniformly in time. The model prediction is shown by the solid curve in the inset histogram, after taking into account the volume surveyed, the finite lifetime of late B-main sequence stars, and assuming the distribution of SMBH-ejection velocities computed by Bromley et al (2006).

The significance of the peak seems high: selecting at random 30 stars from such model yields a peak with 19 (or more) stars in fewer than 1 in 1000 trials. The small number of objects involved precludes a more conclusive assessment of this possibility, but we note that this is not necessarily inconsistent with SMBH ejection. In this scenario, a "preferred travel time" may just reflect a burst of star formation that boosted the population of binaries in the Galactic center region a few hundred Myr ago. We discuss a different interpretation below.

\subsection{HVS angular distribution}

Fig. 2 2 shows an aitoff projection in Galactic coordinates of all stars in the BGKK survey. Highspeed stars (including those reported recently by BGK08) are shown in color, coded according to their travel times, as shown in the bottom-left inset. The main point to note in this figure is that the HVS population is not uniformly distributed in the sky, and that its angular distribution appears inconsistent with a random sample of stars in the survey.

One way of quantifying this is to focus on the region around the constellation of Leo, which seems to contain the majority of HVS. We choose for this a $26^{\circ}$-radius circle centered at the position of Leo I, itself a high-speed (possibly unbound) distant satellite companion of the Milky Way (e.g. Sales et al. 2007a, and Mateo, Olszewski \& Walker 2008). A total of 192 stars in the BGKK survey fall within the circle, or $21 \%$ of the total of 935 halo stars in the northern section of the BGKK survey. By contrast, $42 \%$ of all stars with $V_{r}>280 \mathrm{~km} / \mathrm{s}$ fall within the vicinity of Leo I, a proportion that rises to $54 \%$ for stars with $V_{r}>350 \mathrm{~km} / \mathrm{s}$.

We may estimate the significance of this result by repeating the same calculation after randomly reassigning the measured radial velocities amongst the stars in the survey. A proportion at least as high as $42 \%$ of $V_{r}>280 \mathrm{~km} / \mathrm{s}$ stars falls within 


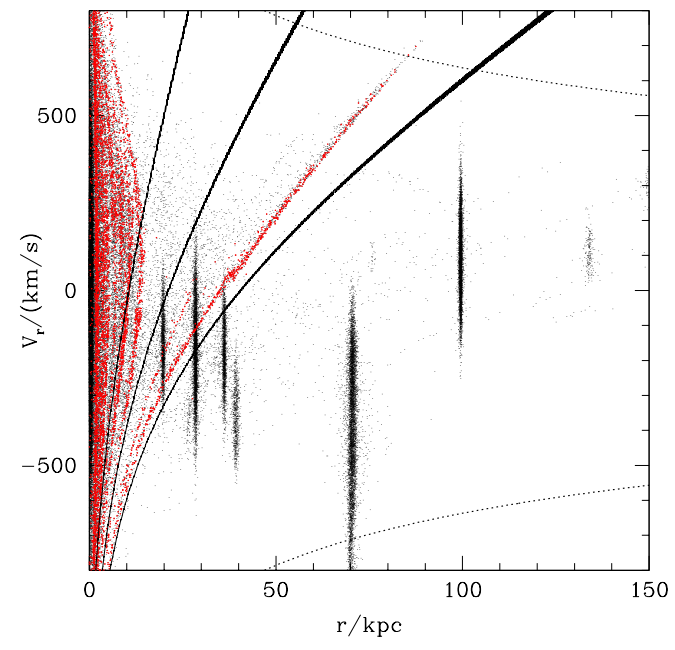

Fig. 3.- Galactocentric radial velocity vs radius for star particles in the simulated galaxy of Abadi et al (2003a,b). Dots in red highlight stars belonging to a satellite that merged recently with the main galaxy. As in Fig. 1, dotted lines show the escape velocity and solid lines denote loci of constant travel time. Note how the tidal debris stripped from the dwarf forms a stream of stars of nearly constant travel time and with velocities which reach and exceed the escape velocity from the system. This shows that hypervelocity stars may also be produced during the disruption of a dwarf galaxy.

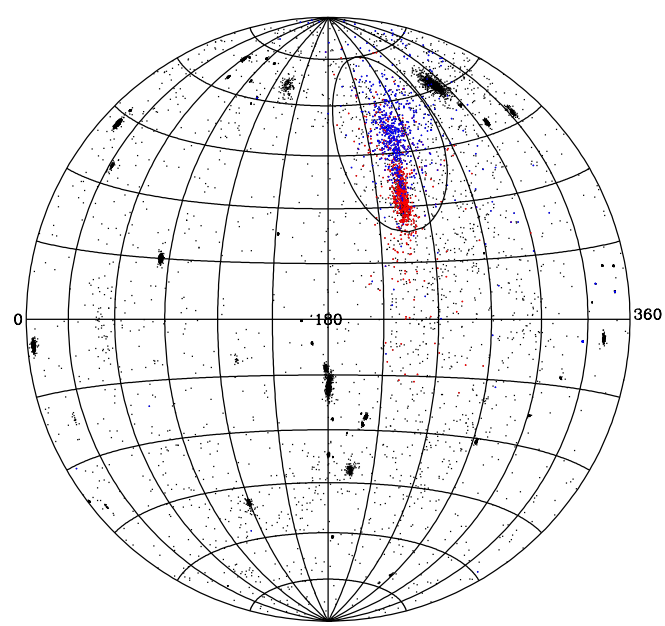

Fig. 4.- Aitoff projection of stars in Fig. 3. after excluding all stars in the main body of the galaxy (i.e., those with $r<20 \mathrm{kpc}$ ), as seen by an observer at the Sun's location. Stars in red are those belonging to the disrupted satellite; those in blue highlight the HVS $\left(V_{r}>400 \mathrm{~km} / \mathrm{s}\right)$ component of the tidal stream. Note that the tidal stream results in an overdensity of high-velocity stars clustered tightly around the location of the tip of the stream and that a gradient in the sky results from the projection of the stream seen nearly "end on". Projected on the sky, the stream spans a few tens of degrees and is roughly fully contained within a $26^{\circ}$-radius circle such as that shown in Fig. 2 . 
the circle in fewer than 1 in 100 such random trials 2 . For $V_{r}>350 \mathrm{~km} / \mathrm{s}$ stars, the corresponding number is fewer than 1 in 125 trials. Also, since the majority of HVS are at Galactic longitude $l \sim 240^{\circ}$ (see Fig. 2) it is unlikely that the HVS anisotropy is the result of the slightly larger effective volume surveyed in the direction of the anti-Galactic center. This supports the idea that the enhanced clustering of HVS in the direction of Leo is real. The observed angular clustering is not easily explained in the SMBH-ejection scenario, which predicts an HVS population distributed approximately isotropically across the sky.

\subsection{A tidal debris origin for hypervelocity stars?}

The anisotropic distribution and the preferred travel time discussed in the preceding subsections suggest that at least part of the HVS population may have a different origin from the SMBHejection mechanism. We explore here an alternative scenario where these two peculiarities are reproduced naturally. Our proposal envisions highvelocity stars in the halo as a result of the tidal disruption of a dwarf galaxy in the Galactic potential.

This is illustrated in Fig. 3, where we show the position of "star" particles in a cosmological simulation of the formation of a galaxy in the $\Lambda \mathrm{CDM}$ cosmogony. The simulation is one in the series presented by Steinmetz and Navarro (2002) and analyzed in detail in Abadi, Navarro \& Steinmetz (2006), where we refer the interested reader to for further technical details. Fig. 3 shows the position of all stars in the $r-V_{r}$ plane, after rescaling the system to a virial velocity of $V_{200}=220 \mathrm{~km} / \mathrm{s}$. This scaling allows us to compare the results directly with observations of the Milky Way. As in Fig. 1, the dotted lines indicate the escape velocity of the system, and the solid curves indicate loci of constant travel time.

The data in Fig. 3 are shown at a snapshot

\footnotetext{
${ }^{2}$ Including the new high-velocity stars identified by BGK08 supports this conclusion. The chance of observing such clustering around Leo I goes down to roughly 1 in 700 for $V_{r}>280 \mathrm{~km} / \mathrm{s}$ stars, and decreases even further if the center of the circle is moved from Leo I in the direction of Leo IV. These estimates assume that the stars in the BGK08 and BGKK parent surveys have similar distributions in the sky.
}

chosen a couple of hundred Myr after the final merger/disruption of a dwarf satellite in the potential of the main galaxy. The stars of the disrupted satellite are shown in red in order to distinguish them from the rest of the stars in the simulated galaxy, shown in black. Note that a long stream of stars formerly belonging to the satellite follow closely a line of constant travel time. These are stars that were stripped from the dwarf during its last pericentric passage, just before its main body merged with the central galaxy. The travel-time coherence in the stream (or "tidal tail") results from the fact that all those stars were stripped from the dwarf at the same time. Stars along the stream have different energies, but it is clear that the disruption event has been able to push some stars into very high-velocity orbits, some even exceeding the local escape speed from the system.

¿From the perspective of an observer near the center of the galaxy (such as an observer at the Sun in the Milky Way) the stream would be seen projected onto a well-defined direction in the sky. This is shown in Fig. 4, which shows a projection on the sky of all stars in the halo of the simulated galaxy (i.e., excluding stars with distances less than $20 \mathrm{kpc}$ from the center). The points in color correspond to the tidal debris from the disrupting dwarf. In particular, blue dots highlight those with radial velocity exceeding $400 \mathrm{~km} / \mathrm{s}$. Note that, from the Sun's perspective, the "tidal tail" of high velocity stars stripped from the dwarf falls within a well-defined region spanning a few tens of degrees in the sky.

Figs. 3 and 4 thus illustrate that tidal disruption of a dwarf galaxy is able to push stars to speeds high enough to escape the galaxy, and provides a mechanism to populate the halo with highvelocity stars with kinematic and angular clustering peculiarities resembling those of the HVS population discussed above. This suggests that at least some stars of the HVS population may have originated in the recent disruption of a dwarf galaxy.

How can this rather unorthodox proposal be validated/ruled out? Fortunately, the most natural predictions of this scenario differ significantly from those motivated by the SMBH-ejection mechanism. In particular, a broader search for highspeed stars should confirm the enhancement of 
HVS in the constellation of Leo. High-speed stars should also exist amongst all spectral types, and should contain a significant number of evolved stars and stars of low metallicity, which should make up the bulk of the stellar content of the dwarf. With enough statistics, a radial-velocity angular gradient amongst high-speed stars might also be observed, such as that shown in Fig. 4. The tail should also contain returning stars with negative velocity that are relatively nearby (see, e.g., Fig. 31) so it would be worth checking existing surveys of nearby stars for unusual velocity patterns in the same general direction in the sky.

We note that our argumentation rests on the assumption that the halo of the Milky Way is relatively massive (i.e., $V_{200} \sim 220 \mathrm{~km} / \mathrm{s}$ ). This is suggested by semianalytic galaxy formation models and is consistent with timing argument mass estimates for the Milky Way (Li \& White 2007). There is evidence, however, that the virial velocity of the Galactic halo might be considerably smaller ( $V_{\text {vir }} \lesssim 140 \mathrm{~km} / \mathrm{s}$ ), as indicated, for example, by recent analysis of Milky Way satellite data (Sales et al 2007b), of RAVE data for solar neighborhood stars (Smith et al 2007), and of SEGUE data on halo stars (Xue et al 2008). This issue remains unresolved, but should a low mass for the Milky Way halo be confirmed, it would mean that a large fraction of HVS would be truly unbound and would argue against the tidal debris interpretation proposed here.

Although examining further a tidal debris origin for HVS appears worthwhile, it should be recognized that this proposal is not without shortcomings. One of the main difficulties is to explain why a tidal stream emanating from a dwarf should contain short-lived, massive main-sequence stars, unless the accreting dwarf was gas-rich and underwent a burst of star formation, perhaps triggered by pericentric passages prior to its final disruption. Gas-rich dwarfs tend to be rather massive, and careful modeling is needed in order to examine whether the timescales really work out and to explain why the remains of the putative dwarf have escaped detection so far. Systematic radialvelocity surveys of large numbers of faint stars across vast regions of the sky, such as those being planned by the GAIA satellite, will undoubtedly be able to settle these questions in the foreseeable future.
MGA acknowledges Laura V. Sales for a careful reading and for comments on an early draft of this paper. We thank the anonymous referee for a constructive report and acknowledge useful discussions with Warren Brown during the KITP Conference "Back to the Galaxy II". This research was supported in part by the National Science Foundation under Grant No. PHY05-51164.

\section{REFERENCES}

Abadi, M. G., Navarro, J. F., Steinmetz, M., \& Eke, V. R. 2003a, ApJ, 591, 499

Abadi, M. G., Navarro, J. F., Steinmetz, M., \& Eke, V. R. 2003b, ApJ, 597, 21

Abadi, M. G., Navarro, J. F., \& Steinmetz, M. 2006, MNRAS, 365, 747

Bromley, B. C., Kenyon, S. J., Geller, M. J., Barcikowski, E., Brown, W. R., \& Kurtz, M. J. 2006, ApJ, 653, 1194

Brown, W. R., Geller, M. J., Kenyon, S. J., \& Kurtz, M. J. 2005, ApJ, 622, L33

Brown, W. R., Geller, M. J., Kenyon, S. J., \& Kurtz, M. J. 2006, ApJ, 647, 303

Brown, W. R., Geller, M. J., Kenyon, S. J., Kurtz, M. J., \& Bromley, B. C. 2007, ApJ, 660, 311

Brown, W. R., Geller, M. J., Kenyon, S. J., Kurtz, M. J., \& Bromley, B. C. 2007, ApJ, 671, 1708

Brown, W. R., Geller, M. J., Kenyon, S. J. 2008, astro-ph/0808.2469

Croton, D. J., et al. 2006, MNRAS, 365, 11

Heber, U., Edelmann, H., Napiwotzki, R., Altmann, M., \& Scholz, R.-D. 2008, A\&A, 483, L21

Hills, J. G. 1988, Nature, 331, 687

Kenyon, S. J., Bromley, B. C., Geller, M. J., \& Brown, W. R. 2008, ApJ, 680, 312

Li, Y.-S., \& White, S. D. M. 2008, MNRAS, 384, 1459

Mateo, M., Olszewski, E. W., \& Walker, M. G. 2008, ApJ, 675, 201 
Navarro, J. F., Frenk, C. S., \& White, S. D. M. 1996, ApJ, 462, 563

Navarro, J. F., Frenk, C. S., \& White, S. D. M. 1997, ApJ, 490, 493

Sales, L. V., Navarro, J. F., Abadi, M. G., \& Steinmetz, M. 2007a, MNRAS, 379, 1464

Sales, L. V., Navarro, J. F., Abadi, M. G., \& Steinmetz, M. 2007b, MNRAS, 379, 1475

Sesana, A., Haardt, F., \& Madau, P. 2007, MNRAS, 379, L45

Smith, M. C., et al. 2007, MNRAS, 379, 755

Xue, X. -., et al. 2008, ArXiv e-prints, 801, arXiv:0801.1232

This 2-column preprint was prepared with the AAS LATEX macros v5.2. 